\title{
CMB polarization features from inflation versus reionization
}

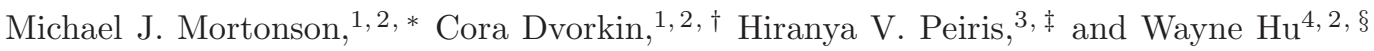 \\ ${ }^{1}$ Department of Physics, University of Chicago, Chicago IL 60637 \\ ${ }^{2}$ Kavli Institute for Cosmological Physics and Enrico Fermi Institute, \\ University of Chicago, Chicago IL 60637, U.S.A. \\ ${ }^{3}$ Institute of Astronomy, University of Cambridge, Cambridge CB3 0HA, U.K. \\ ${ }^{4}$ Department of Astronomy \&s Astrophysics, University of Chicago, Chicago IL 60637
}

(Dated: November 5, 2018)

\begin{abstract}
The angular power spectrum of the cosmic microwave background temperature anisotropy observed by WMAP has an anomalous dip at $\ell \sim 20$ and bump at $\ell \sim 40$. One explanation for this structure is the presence of features in the primordial curvature power spectrum, possibly caused by a step in the inflationary potential. The detection of these features is only marginally significant from temperature data alone. However, the inflationary feature hypothesis predicts a specific shape for the $E$-mode polarization power spectrum with a structure similar to that observed in temperature at $\ell \sim 20-40$. Measurement of the CMB polarization on few-degree scales can therefore be used as a consistency check of the hypothesis. The Planck satellite has the statistical sensitivity to confirm or rule out the model that best fits the temperature features with $3 \sigma$ significance, assuming all other parameters are known. With a cosmic variance limited experiment, this significance improves to $8 \sigma$. For tests of inflationary models that can explain both the dip and bump in temperature, the primary source of uncertainty is confusion with polarization features created by a complex reionization history, which at most reduces the significance to $2.5 \sigma$ for Planck and $5-6 \sigma$ for an ideal experiment. Smoothing of the polarization spectrum by a large tensor component only slightly reduces the ability of polarization to test for inflationary features, as does requiring that polarization is consistent with the observed temperature spectrum given the expected low level of TE correlation on few-degree scales. If polarized foregrounds can be adequately subtracted, Planck will supply valuable evidence for or against features in the primordial power spectrum. A future highsensitivity polarization satellite would enable a decisive test of the feature hypothesis and provide complementary information about the shape of a possible step in the inflationary potential.
\end{abstract}

\section{INTRODUCTION}

Our best constraints on the shape of the primordial power spectrum at large scales come from observations of the cosmic microwave background (CMB) anisotropy by the Wilkinson Microwave Anisotropy Probe (WMAP) [1, 2]. The latest (5-year) WMAP data [3, 4] continue to be well described by the simplest inflationary scenario of a single, slowly rolling, minimally coupled scalar field with a canonical kinetic term [5, 6, 7, 8, 9]. Since the 3year release [10], the WMAP data have indicated a deviation from scale invariance - a red tilt of the scalar spectral index - the significance of which has been debated in the literature from a Bayesian model selection point of view (e.g. [11, 12]). Recent minimally-parametric reconstructions of the primordial power spectrum incorporating some form of penalty for "unnecessary" complexity [13, 14 show some evidence for a red tilt, but no evidence for scale dependence of the spectral index. These methods, as currently implemented, are not very sensitive to sharp, localized features in the primordial power spectrum.

\footnotetext{
*Electronic address: mjmort@uchicago.edu

${ }^{\dagger}$ Electronic address: cdvorkin@uchicago.edu

‡Electronic address: hiranya@ast.cam.ac.uk

$\S$ Electronic address: whu@background.uchicago.edu
}

However, it has been pointed out ever since the original data release [15, 16] that there are several sharp glitches in the WMAP temperature $(T T)$ power spectrum. In particular, several model-independent reconstruction techniques that are sensitive to features localized in a narrow wavenumber range have consistently picked out a feature at $\ell \sim 20-40$ that leads to an improvement of $\Delta \chi^{2} \sim \mathcal{O}(10)$ over a smooth power-law spectrum [17, 18, 19, 20, 21].

Power spectrum features could arise, in principle, in more general classes of inflationary models where slow roll is momentarily violated. Such an effect can be phenomenologically modeled as a discontinuity or singularity in the inflaton potential 22, 23, 24]. A "step-like" feature [23], in particular, would be a good effective field theory description of a symmetry breaking phase transition in a field coupled to the inflaton in multi-field models 25, 26, 27, 28, 29], which can arise in supergravity 30. or M-theory-inspired 31, 32] contexts. Several analyses have confronted such phenomenological descriptions of features in the inflationary potential with current data [16, 33, 34, 35, 36, 37, 38, 39].

It is debatable whether the large scale feature seen in the WMAP TT spectrum is a signal of exotic primordial physics or merely a statistical anomaly. Currently, our information about the smoothness of the primordial power spectrum is dominated by the temperature data. However, future high fidelity CMB polarization measure- 
ments at large scales have the potential to shed light on this question. The importance of polarization data for constraining oscillatory features has been previously discussed in the literature (e.g. 21, 40, 41, 42, 43]) and exploited in particular as a cross-check of the observed low CMB temperature quadrupole [44, 45, 46, 47].

In this work, we propose to use the large-scale polarization of the CMB to test the hypothesis that the $\ell \sim 20-40$ glitch is due to a step in the inflaton potential. We exploit the fact that, in the relevant multipole range, the sharpness of the polarization transfer function and lack of contamination by secondary effects (assuming instantaneous reionization) makes polarization a cleaner probe of such features than temperature [40]. We also investigate how our conclusions are affected by relaxing the assumption of instantaneous reionization [48, 49, 50], changing the parameters of the feature, and including large-amplitude tensor fluctuations. This analysis is particularly timely given the imminent launch of the Planck satellite [51], which promises to greatly increase our knowledge of the large-scale polarization signal. It is also relevant for future dedicated CMB polarization missions [52]. As in a related previous paper on polarization consistency tests of large-angle CMB temperature anomalies [53], it is our objective to make a prediction for the polarization statistics that will be observed by future CMB experiments, given current temperature data, in a "last stand" before Planck.

We present the inflationary model and the numerical procedure used to compute the primordial curvature power spectrum in $\S$ II The polarization consistency tests of the features, both for instantaneous and general reionization histories, are presented in $\S$ III and $\S$ IV and we conclude in $\S \mathbb{\nabla}$. We discuss in Appendix $\AA$ the relation of our work to previous analyses of features in the WMAP temperature data.

\section{INFLATIONARY FEATURES}

We review the inflationary generation of features in the curvature power spectrum from step-like features in the inflaton potential in $₫ \mathrm{IA}$ and their transfer to the CMB temperature and power spectra in $₫ \amalg$

\section{A. Inflationary Model}

To model a feature in the primordial power spectrum that matches the glitches in the WMAP temperature data at $\ell \sim 20-40$, we adopt a phenomenological inflationary potential of the form $V(\phi)=m_{\text {eff }}^{2}(\phi) \phi^{2} / 2$ where the effective mass of the inflaton $\phi$ has a step at $\phi=b$ corresponding to the sudden change in mass during a phase transition [23]:

$$
m_{\mathrm{eff}}^{2}(\phi)=m^{2}\left[1+c \tanh \left(\frac{\phi-b}{d}\right)\right]
$$

with the amplitude and width of the step determined by $c$ and $d$ respectively, assuming that both are positive numbers. We express the potential parameters $m, b$, and $d$ in units of the reduced Planck mass, $M_{\mathrm{Pl}}=(8 \pi G)^{-1 / 2}=$ $2.435 \times 10^{18} \mathrm{GeV}$; the step amplitude $c$ is dimensionless.

In physically realistic models with a sufficiently small step in the potential, the interruption of slow roll as the field encounters the step does not end inflation but affects density perturbations through the generation of scaledependent oscillations that eventually die away. The phenomenology of these oscillations is described in Ref. [23]: the sharper the step, the larger the amplitude and width of the "ringing" superimposed upon the underlying smooth power spectrum. Hence we shall see in $\S \amalg$ IB that lowering $d$ increases the width of the feature in $\ell$ in the CMB power spectra.

Standard slow-roll based approaches are insufficient for computing the power spectrum for this potential, and instead the equation of motion must be integrated numerically mode-by-mode 54]. It is convenient to use the gauge invariant Mukhanov potential [55, 56] for the mode amplitude since it is simply related to the curvature perturbation $\mathcal{R}$ :

$$
u=-z \mathcal{R},
$$

where $z \equiv \dot{\phi} / H, H$ is the Hubble parameter, and the dot denotes a derivative with respect to conformal time. The Fourier components $u_{k}$ obey the equation of motion [57, 58, 59]

$$
\ddot{u}_{k}+\left(k^{2}-\frac{\ddot{z}}{z}\right) u_{k}=0
$$

where $k$ is the modulus of the wavevector $\mathbf{k}$. The power spectrum is defined via the two point correlation function

$$
\left\langle\mathcal{R}_{\mathbf{k}} \mathcal{R}_{\mathbf{k}^{\prime}}^{*}\right\rangle=\frac{2 \pi^{2}}{k^{3}} \Delta_{\mathcal{R}}^{2}(k)(2 \pi)^{3} \delta^{(3)}\left(\mathbf{k}-\mathbf{k}^{\prime}\right),
$$

which is related to $u_{k}$ and $z$ via

$$
\Delta_{\mathcal{R}}^{2}(k)=\frac{k^{3}}{2 \pi^{2}}\left|\frac{u_{k}}{z}\right|^{2} .
$$

The dynamics of the Hubble parameter, described by the Friedmann equation, and the background dynamics of the unperturbed inflaton field, described by the KleinGordon equation, can be written respectively as

$$
\begin{aligned}
& H^{\prime}=-\frac{1}{2} H\left(\phi^{\prime}\right)^{2}, \\
& \phi^{\prime \prime}+\left(\frac{H^{\prime}}{H}+3\right) \phi^{\prime}+\frac{1}{H^{2}} \frac{d V}{d \phi}=0
\end{aligned}
$$

where ${ }^{\prime}=d / d \ln a$. The solution of the mode equation depends on the background dynamics. With the help of these background equations, the mode equation (3) can 


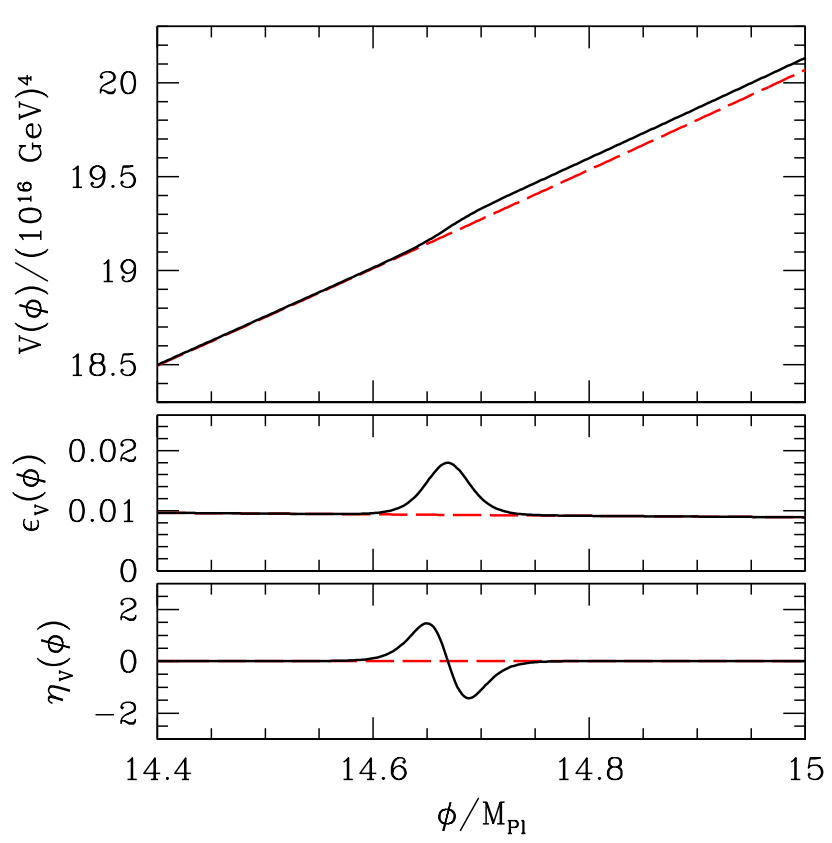

FIG. 1: Upper panel, solid black: Inflationary potential with a step [Eq. 1]. The parameters for the potential are chosen to maximize the WMAP5 likelihood and are listed in Table I The dashed red line shows a smooth $m^{2} \phi^{2}$ potential $(c=0)$ with $m=7.120 \times 10^{-6}$ so that the two models have equal power on small scales $(\phi \ll b)$. Middle and lower panels: slow-roll parameters $\epsilon_{V}$ and $\eta_{V}$ for the two inflationary potentials.

be written as

$$
\begin{gathered}
u_{k}^{\prime \prime}+\left(\frac{H^{\prime}}{H}+1\right) u_{k}^{\prime}+\left\{\frac{k^{2}}{a^{2} H^{2}}-\left[2-4 \frac{H^{\prime}}{H} \frac{\phi^{\prime \prime}}{\phi^{\prime}}\right.\right. \\
\left.\left.-2\left(\frac{H^{\prime}}{H}\right)^{2}-5 \frac{H^{\prime}}{H}-\frac{1}{H^{2}} \frac{d^{2} V}{d \phi^{2}}\right]\right\} u_{k}=0,
\end{gathered}
$$

where the term in square brackets is $\ddot{z} /\left(z a^{2} H^{2}\right)$. We stop integrating the background equations after any transient solution has died away, but while the mode is still well within the horizon. This allows us to obtain initial conditions for the two orthogonal solutions that contribute to $u_{k}$ that are free of contamination due to any transient contribution to the background dynamics. The power spectrum is then obtained by continuing the integration until the mode freezes out far outside the horizon, yielding the asymptotic value of $\left|u_{k} / z\right|$. Further details regarding the numerical solution of the coupled system of differential equations can be found in Ref. 23].

To match a given mode to a physical wavenumber $k$, one must make an assumption about the reheating temperature, but this choice is degenerate with $b$, corresponding to a translation of the step in $\phi$. To compare our results with those of Refs. [33, 34], we adopt the following prescription for the matching:

$$
k_{\star} \equiv a_{\star} H_{\star}=a_{\text {end }} e^{-N_{\star}} H_{\star},
$$

TABLE I: Fiducial feature model parameters chosen to best fit WMAP5 under a flat $\Lambda$ CDM cosmology, compared in the text with a smooth model with $c=0, m=7.120 \times 10^{-6}$, and the same cosmological parameters, which matches the small scale normalization $A_{s}\left(k_{\star}\right)=2.137 \times 10^{-9}$ and tilt $n_{s} \approx 0.96$ at the pivot $k_{\star}=0.05 \mathrm{Mpc}^{-1}$.

\begin{tabular}{cc}
\hline Parameter & Value \\
\hline \hline$m$ & $7.126 \times 10^{-6}$ \\
$b$ & 14.668 \\
$c$ & $1.505 \times 10^{-3}$ \\
$d$ & 0.02705 \\
$N_{\star}$ & 50 \\
\hline$\Omega_{b} h^{2}$ & 0.02238 \\
$\Omega_{c} h^{2}$ & 0.1081 \\
$h$ & 0.724 \\
$\tau$ & 0.089 \\
\hline
\end{tabular}

where $H_{\star}$ is the Hubble scale corresponding to the physical wavenumber $k_{\star}$, which left the horizon $N_{\star} e$-folds before the end of inflation, defined by $d^{2} a / d t^{2}\left(a_{\text {end }}\right)=0$. Following the above authors, we set the pivot scale $k_{\star}=0.05 \mathrm{Mpc}^{-1}$ to correspond to $N_{\star}=50$ (although there are differences in the implementation of the $k$-mode matching that we discuss in Appendix A).

Figure 1 shows our fiducial inflationary potential, with parameters given in Table I that are chosen to fit the WMAP5 temperature glitches at $\ell \sim 20-40$ as we will show in the next section. The number of $e$-folds of inflation after the step in this potential is $N_{\text {step }} \approx 54$. The slow-roll parameters

$$
\epsilon_{V}=\frac{M_{\mathrm{Pl}}^{2}}{2}\left(\frac{d V / d \phi}{V}\right)^{2}, \quad \eta_{V}=M_{\mathrm{Pl}}^{2} \frac{d^{2} V / d \phi^{2}}{V}
$$

are plotted in the lower panels of Fig. [1. Note that near the step at $\phi=b,\left|\eta_{V}\right| \gtrsim 1$ confirming that the slow-roll approximation is not valid. Figure 2 shows the inflationary curvature power spectrum $\Delta_{\mathcal{R}}^{2}(k)$ for this potential, computed by integrating Eqs. (6) - (8) .

For comparison, in Figs. 1 and 2 we also show a smooth, $c=0$ potential with the same small-scale amplitude and tilt as the fiducial potential, and its slow-roll parameters and inflationary power spectrum. The smooth spectrum is nearly indistinguishable from a pure power law of $n_{s} \sim 0.96$ with amplitude $A_{s}\left(k_{\star}\right)=2.137 \times 10^{-9}$. Note that the spectral index is determined by the choices of $N_{\star}$ and $k_{\star}$ in the matching condition of Eq. (9), while the amplitude comes from the inflaton mass $m$. 


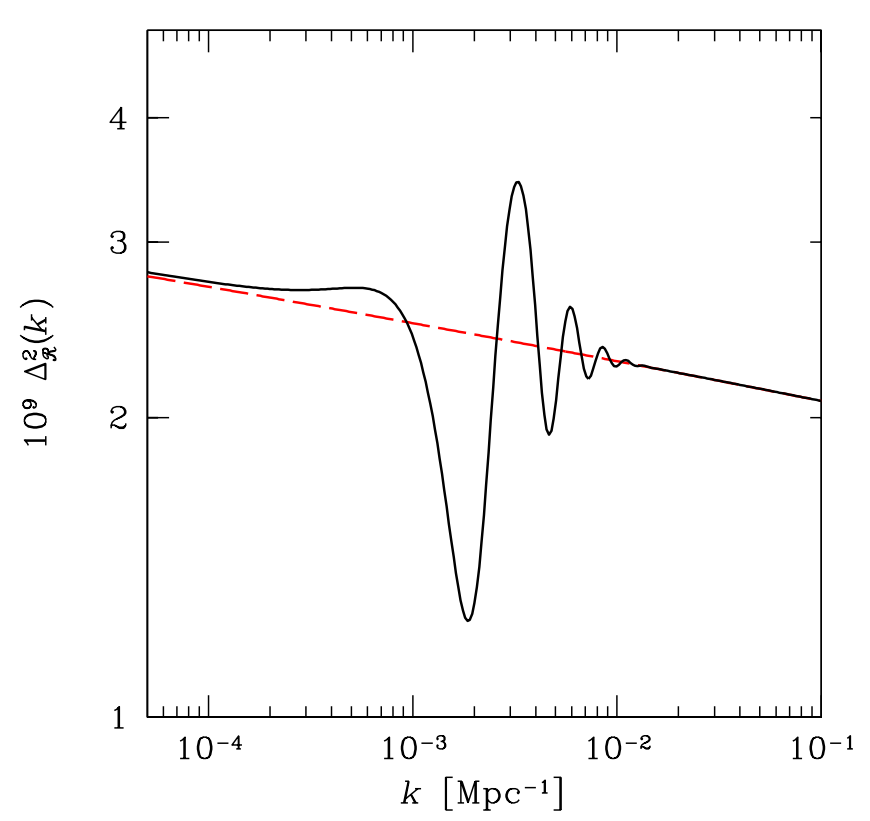

FIG. 2: Primordial curvature power spectra for the potentials in Fig. 1]

\section{B. CMB Power Spectra}

The mapping between the inflationary curvature power spectrum and the observable CMB angular power spectra

$$
\left\langle X_{\ell m}^{*} X_{\ell^{\prime} m^{\prime}}^{\prime}\right\rangle=\delta_{\ell \ell^{\prime}} \delta_{m m^{\prime}} C_{\ell}^{X X^{\prime}},
$$

where $X, X^{\prime} \in T, E$, is given by the scalar radiation transfer functions

$$
\frac{\ell(\ell+1) C_{\ell}^{X X^{\prime}}}{2 \pi}=\int d \ln k T_{\ell}^{X}(k) T_{\ell}^{X^{\prime}}(k) \Delta_{\mathcal{R}}^{2}(k) .
$$

In Fig. 3, we show the $T$ and $E$ transfer functions for the fiducial cosmological parameters of Table [. For a more extended discussion of the transfer functions and their relationship to features in the inflationary power spectrum, see [40]. The resultant temperature and polarization angular power spectra from the inflationary power spectra of Fig. 2 are plotted in Fig. 4.

For the wavenumbers of interest, $1 \lesssim k / 10^{-3} \mathrm{Mpc}^{-1} \lesssim$ 5 , the transfer of power to temperature fluctuations transitions between the Sachs-Wolfe and acoustic regimes at high $\ell$ and carries substantial contributions from the integrated Sachs-Wolfe (ISW) effect at low $\ell$. These effects and geometric projection lead to a very broad mapping of power in $k$ to power in $\ell$. In particular, the oscillations at the upper range in $k$ are largely washed out, leaving only a single broad dip at $\ell \sim 20$ and bump at $\ell \sim 40$ in the temperature spectrum. Likewise, the power at these multipoles correspond to a wide range in $k$ as shown in Fig. [5]

Polarization spectra differ notably from the temperature spectra due to the differences in the transfer function
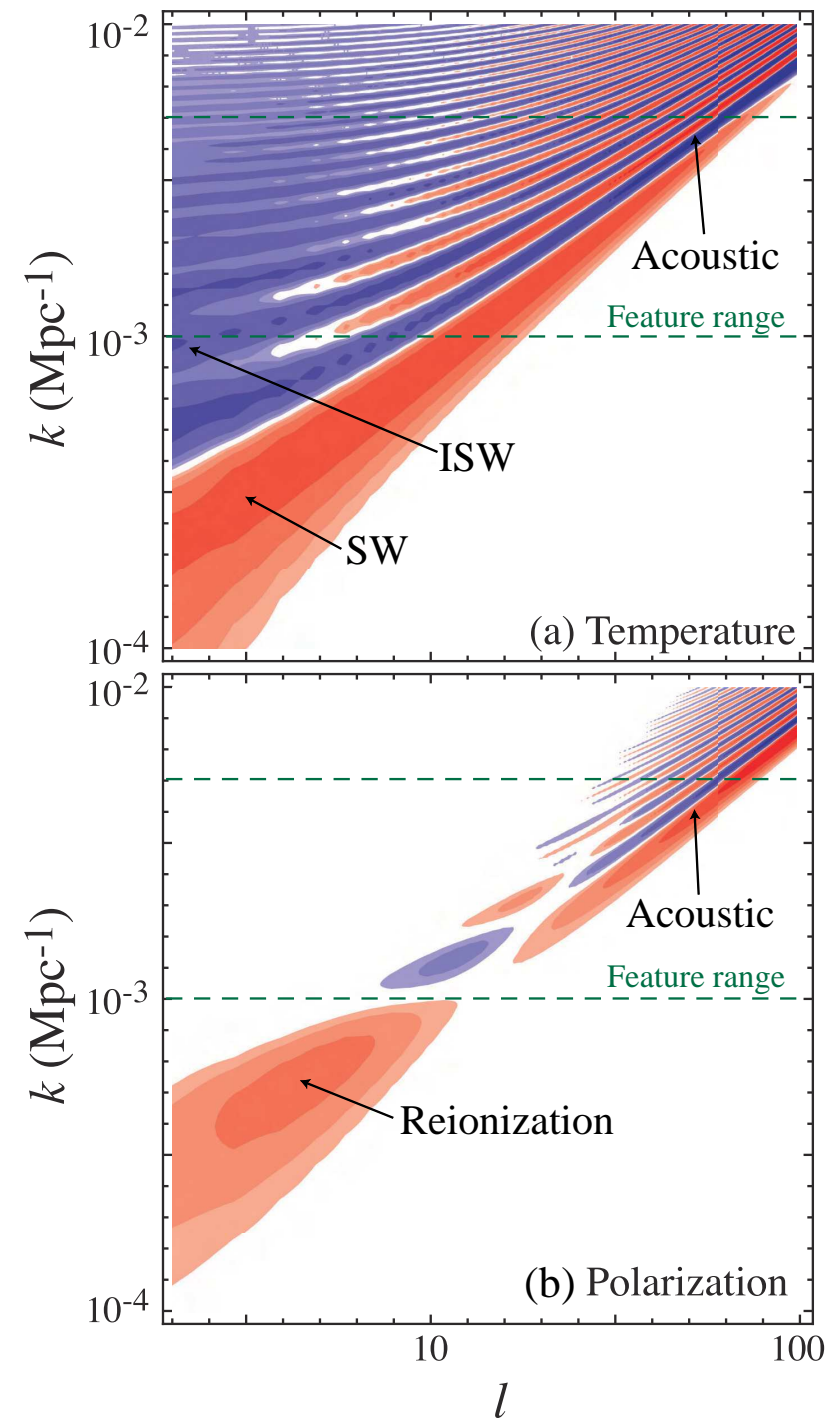

FIG. 3: Transfer function $T_{\ell}^{X}(k)$ for the fiducial model with instantaneous reionization. Upper panel: temperature $X=T$; lower panel: polarization $X=E$. Contours are spaced by factors of 2 . Dashed lines represent the range of $k$-modes where features appear in Fig. 2 Polarization is a cleaner probe of features in this range and, for instantaneous reionization, is nearly uncontaminated by secondary effects. The temperature and polarization are also only weakly correlated here due to the transition between the SachsWolfe (SW) and acoustic regimes in temperature.

shown in Fig. 3. For the standard instantaneous reionization history and the upper portion of the range of $k$ affected by the feature, the polarization is dominated by the onset of acoustic effects only. We shall see that this makes the bump in $\ell \sim 40$ a particularly clean test of inflationary features (see Fig. 5). Furthermore oscillations from high $k$ at higher $\ell$ are retained at a significant level in the polarization.

On the other hand at $k \sim 10^{-3} \mathrm{Mpc}^{-1}$, the polarization transfer from recombination becomes very inefficient and reionization effects come into play. This leads to a very low level of polarization around $\ell \sim 20$ with features 


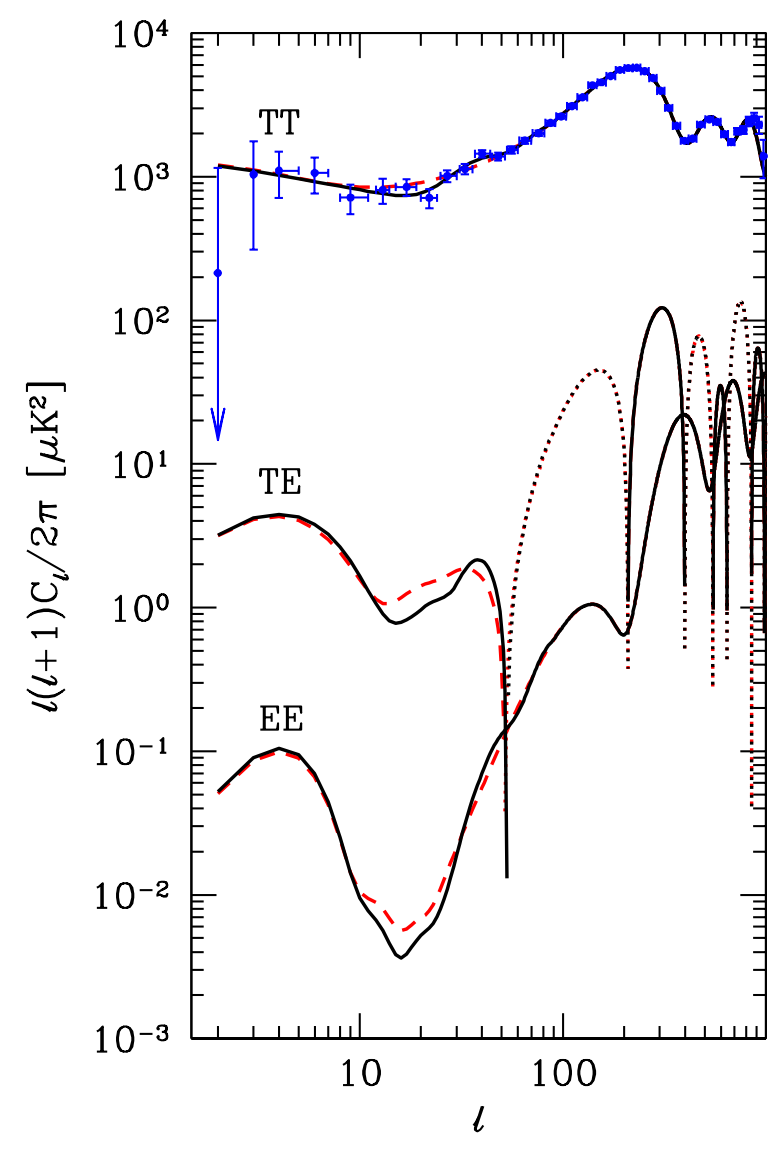

FIG. 4: Temperature and polarization power spectra for the inflationary power spectra in Fig. 2 with solid black curves for the model with a feature and dashed red curves for smooth $\Delta_{\mathcal{R}}^{2}(k)$. Dotted curves indicate where $C_{\ell}^{T E}$ is negative. Blue points with error bars show the 5-year WMAP measurements of $C_{\ell}^{T T}$ including sample variance. For both models, the reionization history is assumed to be instantaneous and the cosmological parameters not determined by the inflationary potential are given in Table $\llbracket$

even for a smooth inflationary power spectrum. These properties leave the $\ell \sim 20$ dip vulnerable to external contamination such as tensor contributions (see $\S$ IIC) or foregrounds as well as uncertainties in the ionization history (see $\S$ IV).

Finally, the cross correlation between the temperature and polarization fields for the entire range of $20 \leq \ell \leq 40$ is very low due to the transition between the Sachs-Wolfe and acoustic-dominated regimes in the temperature field. We shall see in $\S \amalg$ III that this prevents statistical fluctuations in the observed temperature power spectrum from being repeated in the polarization.

These differences in the transfer functions also play a role in defining the region in the potential parameter space that best fits the WMAP $T T$ data versus the region that is best tested by polarization. For the former, we conduct a grid based search over the potential parameters. The mass parameter $m$ determines the amplitude of the spectrum away from the feature and so is mainly fixed by the acoustic peaks at high $\ell$. The location of the

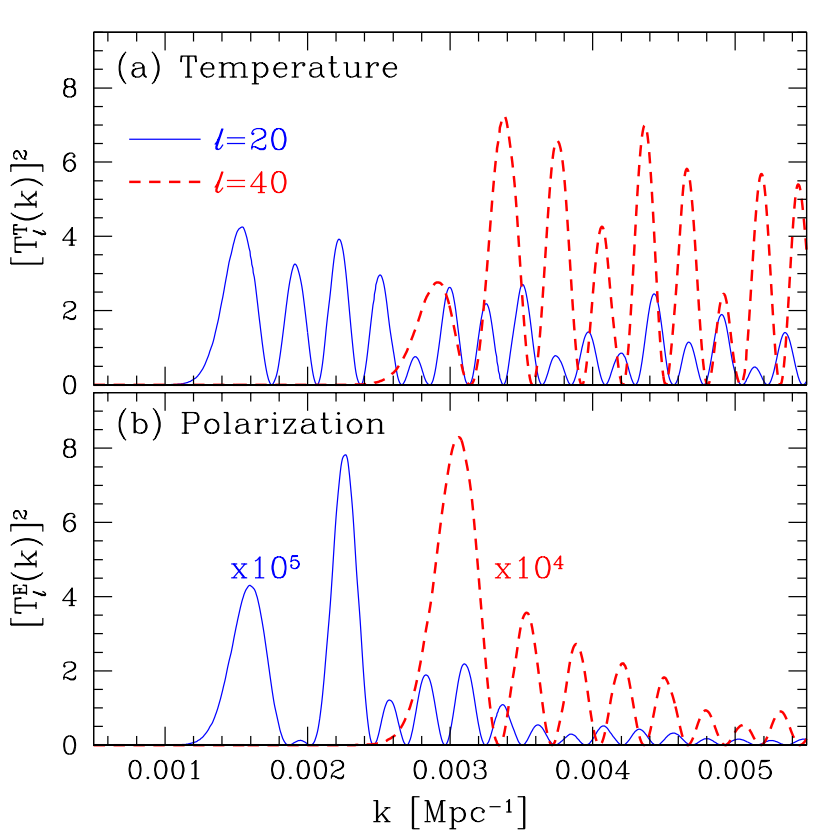

FIG. 5: Transfer function $T_{\ell}^{X}(k)$ for the fiducial model with instantaneous reionization for multipoles near the temperature dip $(\ell=20)$ and bump $(\ell=40)$ for temperature and polarization. For temperature, the dip multipoles receive a broad range of contributions from $k \gtrsim 10^{-3}$ and the bump multipoles from $k \gtrsim 3 \times 10^{-3}$. The localization of the transfer function is sharper for polarization, especially for $\ell=40$ which is immune to reionization effects. The polarization transfer functions have been scaled by $10^{4}$ and $10^{5}$ for convenience.

feature $b$ is also well determined independently of the other parameters [33, 34]. We therefore fix $m$ and $b$ at their best-fit values and search for the best fit in the step amplitude and width parameters $c$ and $d$.

The values of $m, b, c$, and $d$ given in Table \ specify the maximum likelihood model. This model improves the fit to the 5 -year WMAP data by $-2 \Delta \ln \mathcal{L}_{T T} \approx-8$. We will explore variations in the parameters about the maximum and their relationship to the temperature and polarization power spectra through the transfer functions in $\S$ 凹I

The improvement is only marginally significant given the 3 extra parameters of the step and the choice of one out of many possible forms. Matching polarization features can therefore provide a critical confirmation or refutation of the inflationary nature of the temperature features.

\section{CONFIRMING FEATURES WITH POLARIZATION}

In this section, we discuss the significance with which polarization measurements can confirm or rule out the inflationary features discussed in the previous section under the instantaneous reionization model. We begin in $\S \amalg I I A$ with the significance of the best-fit feature model under 
TABLE II: Parameters used when making forecasts for idealized and Planck-like experiments. Here $\Delta_{P}^{(\nu)}$ is in units of $\mu \mathrm{K}$-arcmin.

\begin{tabular}{ccccc}
\hline Experiment & $\nu$ & $\theta_{\mathrm{FWHM}}^{(\nu)}$ & $\Delta_{P}^{(\nu)}$ & $f_{\text {sky }}$ \\
\hline \hline Ideal & - & 0 & 0 & 0.8 \\
\hline Planck & $70 \mathrm{GHz}$ & $14.0^{\prime}$ & 255.6 & 0.8 \\
& $100 \mathrm{GHz}$ & $10.0^{\prime}$ & 109.0 & 0.8 \\
& $143 \mathrm{GHz}$ & $7.1^{\prime}$ & 81.3 & 0.8 \\
\hline
\end{tabular}

the simplest set of assumptions. We assess changes in the significance due to variation in the potential parameters in $\S$ III and due to the inclusion of tensor $E$-modes in $\S$ IIIC In $\S$ IID, we describe the impact of conditioning polarization predictions on the already-measured temperature spectrum.

\section{A. Fiducial Polarization Significance}

To evaluate the significance of discriminating between models, we assume a Gaussian likelihood for the polarization angular power spectrum. In the absence of detector noise, the likelihood $\mathcal{L}_{E E}$ of data $\hat{C}_{\ell}^{E E}$ given a model power spectrum $C_{\ell}^{E E}$ is

$$
-2 \ln \mathcal{L}_{E E} \approx f_{\mathrm{sky}} \sum_{\ell}(2 \ell+1)\left(\frac{\hat{C}_{\ell}^{E E}}{C_{\ell}^{E E}}+\ln \frac{C_{\ell}^{E E}}{\hat{C}_{\ell}^{E E}}-1\right),
$$

where $f_{\text {sky }}$ is the fraction of sky with usable $E$ measurements. If the data $\hat{C}_{\ell}^{E E}$ have no inflationary feature and the model $C_{\ell}^{E E}$ has the inflationary feature, we call this the significance at which false positives can be rejected. Conversely, if the data have an inflationary feature and the model spectrum has no feature, we call this the significance at which false negatives can be rejected.

For forecasts throughout this paper, we assume that the data are equal to the ensemble average of realizations for a particular model. Therefore, the minimum $-2 \ln \mathcal{L}$ is zero and $-2 \Delta \ln \mathcal{L}=-2 \ln \mathcal{L}$. The exception to this is that when we discuss the WMAP TT likelihood, the relevant quantity is $-2 \Delta \ln \mathcal{L}=-2 \ln \left(\mathcal{L} / \mathcal{L}_{\mathrm{ML}}\right)$ where the likelihood of the best fit model is $-2 \ln \mathcal{L}_{\mathrm{ML}} \neq 0$. We make forecasts for an ideal, sample variance limited experiment and for Planck using the experimental specifications in Table I. For the Planck case with a finite noise power $N_{\ell}, C_{\ell} \rightarrow C_{\ell}+N_{\ell}$ in Eq. (13), where $N_{\ell}$ is the minimum variance combination of the noise powers of the individual frequency channels

$$
N_{\ell}^{(\nu)}=\left(\frac{\Delta_{P}^{(\nu)}}{\mu \mathrm{K}-\mathrm{rad}}\right)^{2} \exp \left[\frac{\ell(\ell+1)\left(\theta_{\mathrm{FWHM}}^{(\nu)} / \mathrm{rad}\right)^{2}}{8 \ln 2}\right]
$$

using $\Delta_{P}^{(\nu)}$ and $\theta_{\mathrm{FWHM}}^{(\nu)}$ from Table $\amalg$ converted to the appropriate units.
TABLE III: $-2 \Delta \ln \mathcal{L}_{E E}$ for false positive and false negative tests comparing models with smooth $\Delta_{\mathcal{R}}^{2}(k)$ and a feature in $\Delta_{\mathcal{R}}^{2}(k)$.

\begin{tabular}{llr}
\hline Experiment & Test & $-2 \Delta \ln \mathcal{L}_{E E}$ \\
\hline \hline Ideal & False positive & 64 \\
Ideal & False negative & 60 \\
\hline Planck & False positive & 8 \\
Planck & False negative & 9 \\
\hline
\end{tabular}

Table III lists $-2 \Delta \ln \mathcal{L}_{E E}$ for rejecting false positives and false negatives. The significance of false positive or negative rejection in this most optimistic case is $\sqrt{-2 \Delta \ln \mathcal{L}_{E E}} \sim 8$ for the ideal experiment and $\sim 3$ for Planck. In the following sections, we will discuss various effects that can degrade this significance.

\section{B. Potential Parameters}

Variation in the parameters of the inflationary potential from the best fit model can affect the significance of polarization tests of features. As noted in $\S \llbracket \mathrm{B}, m$ and $b$ are strongly constrained by the observed CMB temperature spectrum, but the parameters $c$ and $d$ that control the amplitude and width of an inflationary step are less well determined by temperature alone.

In terms of the curvature power spectrum, increasing $c$ increases the amplitude of the features. However, decreasing the width of the potential step by lowering $d$ enhances the deviations from slow roll, thereby also amplifying the feature in the power spectrum.

Figure 6 shows a contour plot of the WMAP temperature likelihood $-2 \Delta \ln \mathcal{L}_{T T}$ for the parameters $c$ and $d$

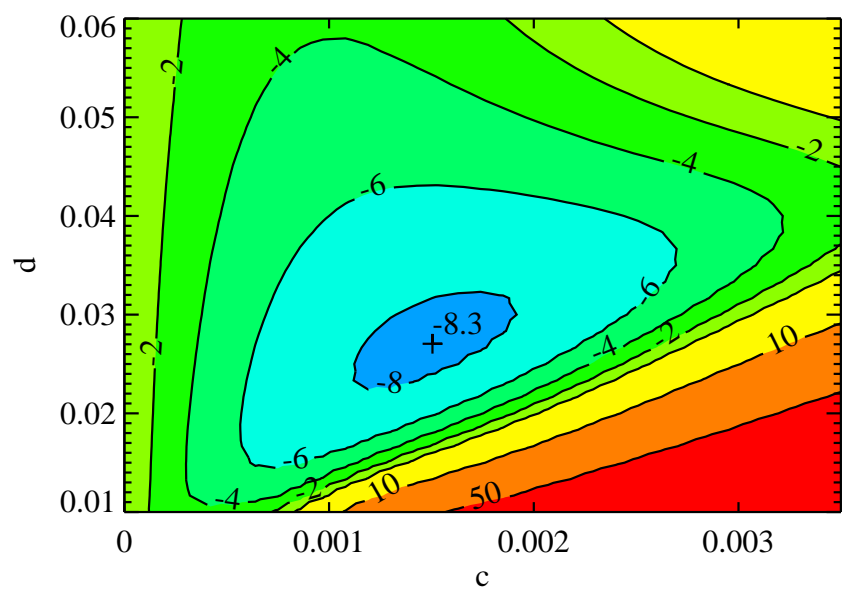

FIG. 6: Contour plot of $-2 \Delta \ln \mathcal{L}_{T T}$ for parameters $c$ and $d$ using 5-year WMAP data. Other potential parameters are fixed at their fiducial values. The minimum, with $-2 \Delta \ln \mathcal{L}_{T T}=-8.3$ relative to the smooth $c=0$ model, is shown with a cross. 

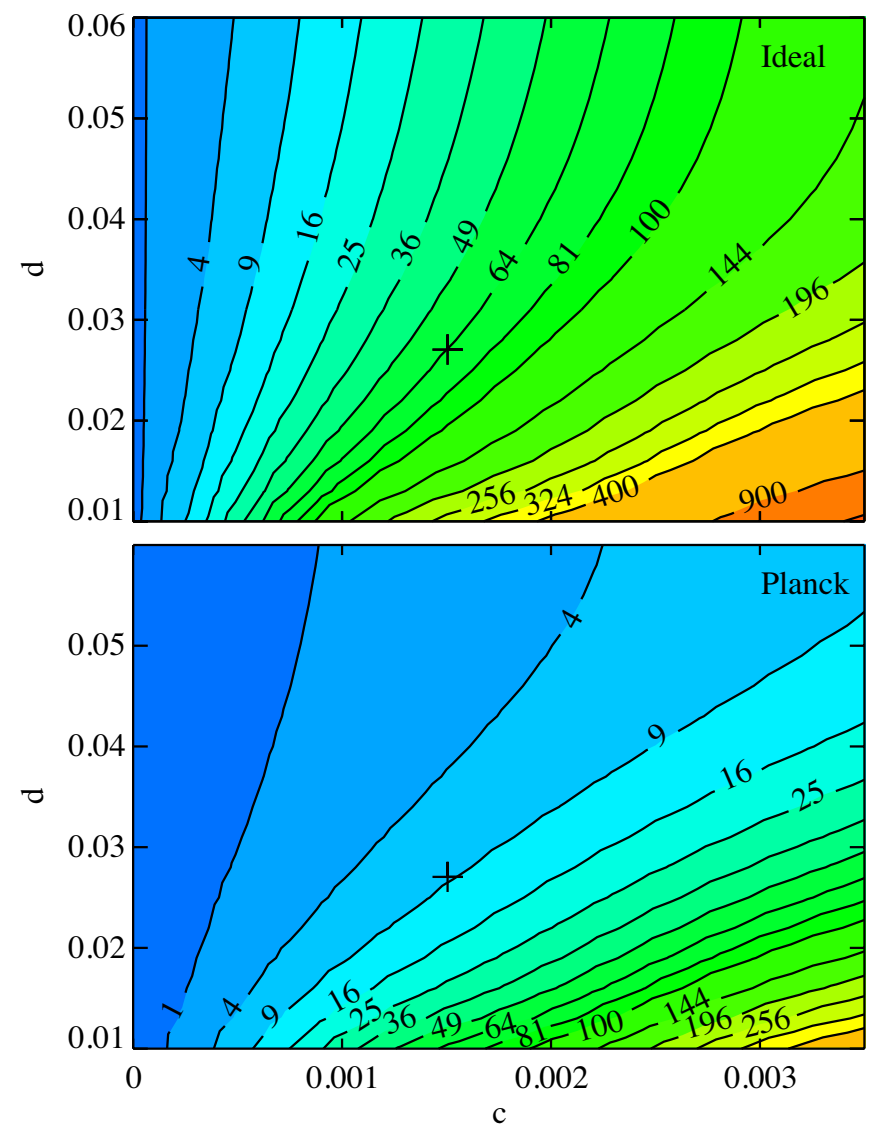

FIG. 7: Contour plot of $-2 \Delta \ln \mathcal{L}_{E E}$ for the parameters $c$ and $d$, for tests of false positives with a cosmic variance limited experiment (upper panel) and Planck (lower panel). The best fit model to WMAP TT is shown with a cross.

(relative to $c=0$ ) and Fig. 7 shows $-2 \Delta \ln \mathcal{L}_{E E}$ for false positives using simulated polarization data. The similarities and differences between these two plots reflect properties of the temperature and polarization transfer functions.

For the temperature case near the minimum, the degeneracy between the two parameters is approximately $c \propto d^{2}$. This line roughly corresponds to keeping the amplitude of the enhanced power in $\Delta_{\mathcal{R}}^{2}(k)$ at $k \sim$ $3 \times 10^{-3} \mathrm{Mpc}^{-1}$ fixed. The preferred value of $c$ corresponds to the best amplitude of the negative dip at $k \sim 2 \times 10^{-3} \mathrm{Mpc}^{-1}$. For the best fit parameters, including the feature in $\Delta_{\mathcal{R}}^{2}(k)$ improves the fit to 5 -year WMAP data by $-2 \Delta \ln \mathcal{L}_{T T} \approx-8$.

Due to the weak significance of the feature detection, the contours become substantially distorted away from the maximum likelihood. In particular, the contours in Fig. [6 show a triangular region extending to high $d \sim$ 0.04. This region corresponds to a lower amplitude in both the first dip and bump in $k$ as shown in Fig. 8, Due to projection effects in temperature, the $\ell=20$ dip gets contributions from both the dip and the bump in $k$ (see Fig. (5). Consequently, a model with smaller features in $k$ in both the dip and bump can lead to the same amplitude

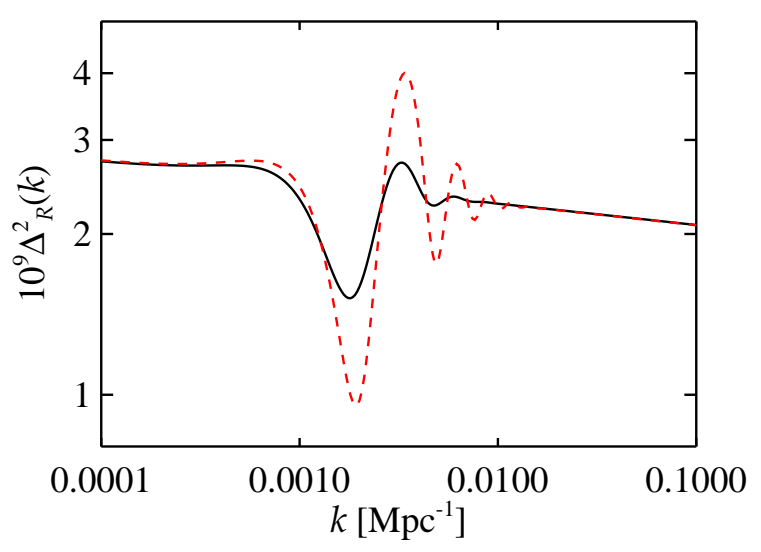

FIG. 8: Primordial curvature power spectra for models illustrating projection degeneracies in the temperature. The parameters of the two models are chosen to have similar temperature dips at $\ell \sim$ 20 and equal WMAP likelihoods: $(c, d)=(0.00128,0.043)$ ( solid black) and $(c, d)=(0.0023,0.028)$ (dashed red); other parameters are fixed to the values in Table $\prod$ for both models.

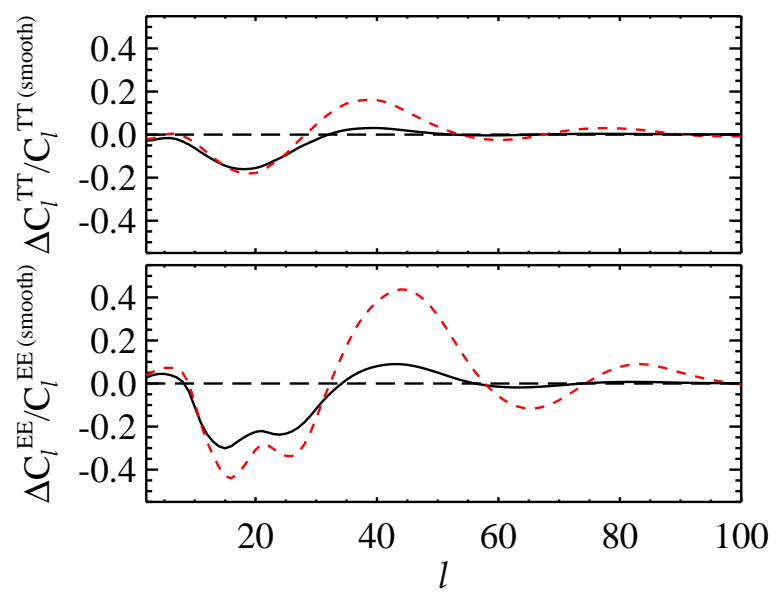

FIG. 9: Upper panel: Relative difference in $C_{\ell}^{T T}$, with respect to a smooth power spectrum, of two models with equal $T T$ amplitude at $\ell \sim 20$ and curvature power spectra shown in Fig. 8 Lower panel: Due to differences in the polarization transfer function, the models do not have degenerate $\ell \sim 20$ dips and show significant differences at $\ell \gtrsim 60$ as well, leading to a much higher polarization significance for the model plotted with dashed lines.

of the dip at $\ell=20$ if the amplitude of the bump is reduced more.

We illustrate these projection effects in Figs. 8 and 9 with two models chosen to have the same likelihood improvement of $-2 \Delta \ln \mathcal{L}_{T T} \approx-6$. For the model with smaller features in $k$, the temperature enhancement at $\ell \sim 40$ is substantially reduced compared with the bestfit model, while the model with larger features in $k$ overshoots the bump at $\ell=40$ in temperature. Despite these differences, both models have about the same $T T$ amplitude in the $\ell=20$ dip as the best-fit model but a slightly worse overall fit. In particular, for the model with smaller 
features in $k$, inflationary features can only explain the observed $\ell=20 \mathrm{dip}$ in temperature and not the $\ell=40$ bump.

The degeneracies in $c$ and $d$ for polarization significance share similarities with, yet have important differences from, those for temperature. The polarization significance remains largely unchanged for small variations in $c$ and $d$ along the constant $c / d^{2}$ line favored by the temperature spectra. Near the maximum, variations along this direction preserve the amplitude of intrinsic features in $k$ (see Fig. 6). However within the $-2 \Delta \ln \mathcal{L}_{T T}=-4$ region the significance for a ideal experiment can either drop or rise significantly. The reason is that due to projection effects in temperature, the polarization better separates changes in the overall and relative amplitude of the features in $k$. In the triangular high $d$ region, where the amplitude of the $T T$ dip remains unchanged but the intrinsic features in $k$ are all reduced, the significance of the polarization difference decreases markedly (see Fig. 9). Because of the sharper projection, even the $\ell \sim 20$ dip in polarization is reduced. The net result is that the polarization significance is a stronger function of $c$, which controls the overall amplitude, than the temperature significance.

Note that while the significance can be substantially degraded from our best fit assumptions, this is mainly because of the weak detection of a feature in the temperature spectrum itself. In cases where the polarization significance is greatly reduced, the temperature bump at $\ell \sim 40$ cannot be explained by the inflationary features. In other words, polarization remains a robust probe of the inflationary nature of the $\ell=40$ bump across variations of the potential parameters.

\section{Tensors}

The $m^{2} \phi^{2}$ potential with the parameters in Table凹predicts substantial gravitational wave contributions with tensor-to-scalar ratio $r \approx 0.16$. Relative to a smooth$\Delta_{\mathcal{R}}^{2}(k)$ model without tensors, the $m^{2} \phi^{2}$ model with a feature has extra distinguishing power due to the presence of $B$-mode polarization. Because other forms for the potential can also be used as the smooth base on which to place the feature 34] we choose not to include tensors for most of our calculations. Moreover, a $B$-mode detection would not be useful for discriminating features. The $B$-mode amplitude is insensitive to features since the potential amplitude is left nearly unchanged by the step. Additionally, a small step in the potential is not expected to generate features in the CMB power spectra of tensor modes. Unlike the scalar spectrum whose shape is sensitive to the second slow-roll parameter $\eta_{V}$, the shape of the tensor spectrum depends primarily on $\epsilon_{V}$, which remains small at the step (see Fig. 1, 34]). In Fig. 10 we show the $B$-mode prediction for $r=0.16$ and a pure power law tensor spectrum with tilt $n_{t}=-r / 8$.

On the other hand, it is important to assess the possi-

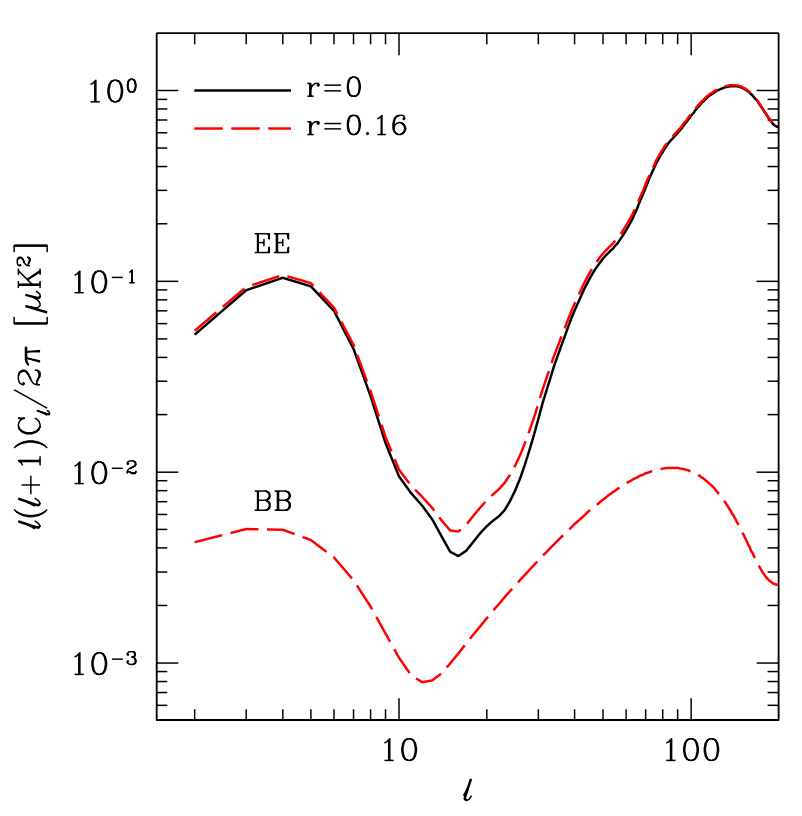

FIG. 10: Effect of tensor fluctuations on polarization power spectra for the model with a feature in $\Delta_{\mathcal{R}}^{2}(k)$. Solid black: no tensor component. Dashed red: including tensors with $r=0.16$. Tensors smooth the $E E$ spectrum near the $\ell \sim 20$ dip.

bility of degradation of the $E$-mode feature from the curvature spectrum due to the nearly smooth tensor $E$-mode contributions. Due to the shape of the tensor $E$-mode spectrum, which mimics the $B$-mode spectrum, the main impact of tensors is to fill in the dip in the polarization spectrum around $\ell \sim 20$ (see Fig. 10). Correspondingly, the decrease in significance for the ideal experiment is $13-15 \%$ in $\sqrt{-2 \Delta \ln \mathcal{L}_{E E}}$, and for Planck, 4\%. Planck is less affected since its lower sensitivity limits the accuracy of measurements in the $\ell \sim 20$ dip. Since these degradations are relatively small, we ignore tensors when considering the impact of the reionization history below. Moreover, we shall see that reionization uncertainties are very similar to tensors in that they make the $\ell \sim 20$ dip less useful for distinguishing features through $E$-mode polarization.

\section{Temperature Conditioning}

The usefulness of polarization for providing an independent test of features observed in temperature may also be reduced by the correlation of temperature and polarization: a positive correlation would make observation of polarization features more likely given the WMAP TT data regardless of whether the features have an inflationary or chance statistical origin. We expect the reduction in significance to be small given that $C_{\ell}^{T E}$ is small on the relevant angular scales (see Fig. (4), and in this section we quantify this statement. 
To assess the impact of conditioning polarization predictions on the WMAP temperature data, it is convenient to replace the likelihood statistic of Eq. (13) with a $\chi^{2}$ statistic. This allows us to phrase the impact in terms of the bias and change in variance predicted for the $E E$ power spectrum from the $T T$ measurements. Note that in the absence of the temperature constraint and in the limit of small differences between the model and the data,

$$
\begin{aligned}
-2 \Delta \ln \mathcal{L}_{E E} & \approx f_{\text {sky }} \sum_{\ell} \frac{2 \ell+1}{2}\left(\frac{\hat{C}_{\ell}^{E E}-C_{\ell}^{E E}}{C_{\ell}^{E E}}\right)^{2} \\
& \approx \sum_{\ell} \frac{\left(\hat{C}_{\ell}^{E E}-C_{\ell}^{E E}\right)^{2}}{\operatorname{Var}\left(\hat{C}_{\ell}^{E E}\right)}
\end{aligned}
$$

which is equal to a simple $\chi^{2}$ statistic.

Now let us include the temperature constraint. First take the idealization that the temperature multipole moments $T_{\ell m}$ have been measured on the full sky with negligible noise. Given a model that correlates the polarization field through the cross correlation coefficient

$$
R_{\ell}=\frac{C_{\ell}^{T E}}{\sqrt{C_{\ell}^{T T} C_{\ell}^{E E}}},
$$

a constrained realization of the polarization field that is consistent with the temperature field can be constructed as

$$
\frac{E_{\ell m}}{\sqrt{C_{\ell}^{E E}}}=R_{\ell} \frac{T_{\ell m}}{\sqrt{C_{\ell}^{T T}}}+\sqrt{1-R_{\ell}^{2}} g_{\ell m},
$$

where $g_{\ell m}$ is a complex Gaussian field with zero mean, unit variance $\left\langle g_{\ell m} g_{\ell m}^{*}\right\rangle=1$, and a real transform $g_{\ell m}^{*}=$ $(-1)^{m} g_{\ell,-m}$. The estimate of the power spectrum is then

$$
\hat{C}_{\ell}^{E E}=\frac{1}{2 \ell+1} \sum_{m} E_{\ell m}^{*} E_{\ell m}
$$

and its mean over the constrained realizations is biased from the true $C_{\ell}^{E E}$

$$
\frac{\left\langle\hat{C}_{\ell}^{E E}\right\rangle-C_{\ell}^{E E}}{C_{\ell}^{E E}}=R_{\ell}^{2} \frac{\hat{C}_{\ell}^{T T}-C_{\ell}^{T T}}{C_{\ell}^{T T}},
$$

by the fixed observed temperature power spectrum $\hat{C}_{\ell}^{T T}=\sum_{m} T_{\ell m} T_{\ell m}^{*} /(2 \ell+1)$. With a high correlation coefficient, chance features in the temperature spectrum induce similar features in the observed polarization spectrum. For example, if $T T$ fluctuates high, $E E$ will also fluctuate high (on average).

The temperature constraint also removes some of the freedom in the variance of the polarization power spectrum:

$$
\begin{aligned}
\frac{\operatorname{Var}\left(\hat{C}_{\ell}^{E E}\right)}{\left(C_{\ell}^{E E}\right)^{2}} & =\frac{2}{2 \ell+1}\left(1-R_{\ell}^{2}\right)^{2} \\
& +\frac{4}{2 \ell+1} R_{\ell}^{2}\left(1-R_{\ell}^{2}\right) \frac{\hat{C}_{\ell}^{T T}}{C_{\ell}^{T T}} .
\end{aligned}
$$

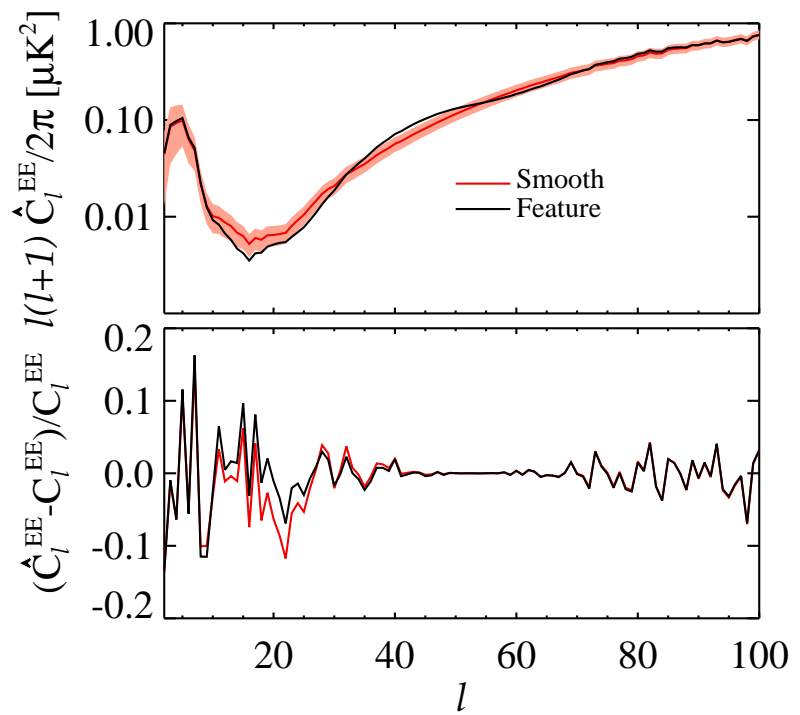

FIG. 11: Upper panel: Solid lines show the $E$-mode power spectrum constrained to the temperature data for the smooth- $\Delta_{\mathcal{R}}^{2}(k)$ model along with the band representing sample variance per $\ell$ for the ideal experiment. The model with a feature (dashed) lies significantly outside of the band in the $10 \lesssim \ell \lesssim 60$ range, making false negatives unlikely. Lower panel: Fractional difference between the average of the constrained realizations $\left\langle\hat{C}_{\ell}^{E E}\right\rangle$ and the full ensemble average $C_{\ell}^{E E}$ for both models. The impact of the constraint is minimal due to the lack of correlation between the temperature and polarization fields in the region of interest.

In the limit that the correlation $R_{\ell} \rightarrow 0$, the variance takes on its usual form for a Gaussian random field. In the limit that $R_{\ell} \rightarrow 1$, there is no uncorrelated piece and the observed temperature spectrum determines the observed polarization spectrum with no variance.

Now let us add in detector noise and finite sky coverage. Given a noise power spectrum $N_{\ell}^{E E}$ and a fraction of the sky $f_{\text {sky }}$,

$$
\begin{array}{r}
f_{\mathrm{sky}} \frac{\operatorname{Var}\left(\hat{C}_{\ell}^{E E}\right)}{\left(C_{\ell}^{E E}\right)^{2}} \approx \frac{2}{2 \ell+1}\left(1-R_{\ell}^{2}+\frac{N_{\ell}^{E E}}{C_{\ell}^{E E}}\right)^{2} \\
+\frac{4}{2 \ell+1} R_{\ell}^{2}\left(1-R_{\ell}^{2}+\frac{N_{\ell}^{E E}}{C_{\ell}^{E E}}\right) \frac{\hat{C}_{\ell}^{T T}}{C_{\ell}^{T T}} .
\end{array}
$$

Figure 11 shows, in the upper panel, the $E$-mode polarization power spectrum for the smooth inflationary spectrum constrained to WMAP5 temperature data for the ideal experiment. For comparison, $C_{\ell}^{E E}$ for the best fit feature model is also plotted. Note that even the second dip in the spectrum at $\ell \sim 60$ remains significantly distinct in polarization. In the lower panel, the impact of the temperature power spectrum constraint is plotted as the fractional difference between $\hat{C}_{\ell}^{E E}$ and $C_{\ell}^{E E}$ for each model. Due to the lack of temperature-polarization correlation in the $10 \lesssim \ell \lesssim 60$ regime, the impact of the constraint on the polarization features is negligible.

We can quantify these conclusions by generalizing the $\chi^{2}$ statistic in Eq. (15) to include the temperature con- 
TABLE IV: $\Delta \chi_{E E}^{2}$ for false positive and false negative tests comparing models with smooth $\Delta_{\mathcal{R}}^{2}(k)$ and a feature in $\Delta_{\mathcal{R}}^{2}(k)$, with polarization either unconstrained or constrained to observed temperature data.

\begin{tabular}{llrr}
\hline & & \multicolumn{2}{c}{$\Delta \chi_{E E}^{2}$} \\
Experiment & Test & w/o $T$ & with $T$ \\
\hline \hline Ideal & False positive & 70 & 63 \\
Ideal & False negative & 59 & 56 \\
\hline Planck & False positive & 8 & 8 \\
Planck & False negative & 9 & 8 \\
\hline
\end{tabular}

straint:

$$
\Delta \chi_{E E}^{2} \equiv \sum_{\ell} \frac{\left(\hat{C}_{\ell}^{E E}-\left\langle\hat{C}_{\ell}^{E E}\right\rangle\right)^{2}}{\operatorname{Var}\left(\hat{C}_{\ell}^{E E}\right)}
$$

As in the likelihood analysis, we assume that the data are a typical draw of the true model ("1") and that we are testing the significance at which the second model ("2") can be rejected. Then we set $\hat{C}_{\ell}^{E E}=\left\langle\hat{C}_{\ell}^{E E(1)}\right\rangle,\left\langle\hat{C}_{\ell}^{E E}\right\rangle=$ $\left\langle\hat{C}_{\ell}^{E E(2)}\right\rangle$, and $\operatorname{Var}\left(\hat{C}_{\ell}^{E E}\right)=\operatorname{Var}\left(\hat{C}_{\ell}^{E E(2)}\right)$. Note that the bias induced by the temperature constraint enters into both models whereas the change in the variance enters only from model 2 .

Table IV assesses the $\chi^{2}$ significance of the rejection of false positives and false negatives for the fiducial feature model. In the last column we have applied the constraint from the WMAP5 temperature data and in the penultimate column we artificially drop the constraint by setting $R_{\ell}=0$ in the evaluation of $\Delta \chi_{E E}^{2}$. Even with the constraint, the significance with which false positives can be rejected is $\sqrt{\Delta \chi_{E E}^{2}}=7.9$ for the ideal experiment and $\sqrt{\Delta \chi_{E E}^{2}}=2.8$ for Planck. For the case of false negatives, these numbers become 7.5 for the ideal experiment and 2.8 for Planck. The difference between false positive and false negative significances comes from the dependence of sample variance on the model tested. In all cases, the significance in terms of $\Delta \chi_{E E}^{2}$ is comparable to $-2 \Delta \ln \mathcal{L}_{E E}$ in Table III

The impact of the temperature constraint is to lower the significance of both cases but only by $\lesssim 5 \%$ in $\sqrt{\Delta \chi_{E E}^{2}}$. This small difference in significance justifies our choice to omit the constraint to temperature in our exploration of other effects that can degrade the significance.

\section{REIONIZATION FEATURES}

A more complicated ionization history can in principle produce features in the polarization spectrum that might mimic or obscure features from the inflationary power spectrum. This is especially true for the dip at $\ell \sim 20$.
TABLE V: $-2 \Delta \ln \mathcal{L}_{E E}$ for tests of false positives and false negatives with ionization histories of the data and model tuned at $6<z<50$ to minimize the significance of rejection using the methods described in $\S$ IVB

\begin{tabular}{llr}
\hline Experiment & Test & $-2 \Delta \ln \mathcal{L}_{E E}$ \\
\hline \hline Ideal & False positive & 36 \\
Ideal & False negative & 25 \\
\hline Planck & False positive & 6 \\
Planck & False negative & 6 \\
\hline
\end{tabular}

In this section we search for ionization histories that lead to a higher incidence of false positives and false negatives.

Reduced significance of false positives or negatives due to confusion between features from inflation and from reionization can arise in two ways. First, the true reionization history can introduce features in the data that either falsely mimic inflationary features or hide true features. Second, additional reionization freedom in the (false) model we wish to test can allow a better match to data generated from the alternate (true) model assumption. To account for both effects, we use a two-step method in which we first optimize the ionization history of the true model to produce a false positive or negative result, and then vary the ionization history of the false model. We will describe this procedure in $\S$ IV B.

Table $\mathrm{V}$ summarizes the results of this section. Relative to the significance of rejecting false positives or negatives for instantaneous reionization models, ionization freedom lowers the significance for an ideal experiment by a factor of $0.64-0.75$ to $\sqrt{-2 \Delta \ln \mathcal{L}_{E E}} \approx 5-6$, and for Planck by a substantially smaller factor of $0.83-0.87$ to $\sqrt{-2 \Delta \ln \mathcal{L}_{E E}} \approx 2.5$. Given the amount of freedom we allow in the ionization history these should be viewed as the maximal degradation possible due to reionization. We describe the details of this calculation in the following sections.

\section{A. Reionization Principal Components}

The form of the ionization history, and therefore the shape of the large-scale reionization peak in the polarization spectrum, are only weakly constrained by current observations and theoretical modeling, especially on the scales relevant for inflationary features [60]. We treat the evolution of the mean ionized fraction of hydrogen with redshift, $x_{e}(z)$, as an unconstrained function between $z=6$ and some high redshift $z=z_{\max }$. At lower redshifts, we assume $x_{e} \approx 1$ as required by the observed Ly $\alpha$ transmission in quasar spectra at $z \lesssim 6$ (see e.g. 61]). The highest redshift of reionization is less certain, so we take $z_{\max }=50$ which is quite conservative for conventional sources of ionizing radiation.

We parametrize general reionization histories with a 
basis of principal components (PCs) $S_{i}(z)$ of the largescale $E$-mode polarization [48]. We use the 7 lowestvariance $\mathrm{PCs}$ only since the higher-variance $\mathrm{PCs}$ have a negligible impact on the polarization power spectrum. Thus the ionization history at $6<z<50$ is

$$
x_{e}(z)=x_{e}^{\mathrm{fid}}+\sum_{i=1}^{7} m_{i} S_{i}(z),
$$

where we take a constant fiducial ionized fraction of $x_{e}^{\mathrm{fid}}=0.07$ so that the fiducial model with $\left\{m_{i}\right\}=0$ has a total reionization optical depth of $\tau \approx 0.09$.

We vary the $\mathrm{PC}$ amplitudes $\left\{m_{i}\right\}$ and compare the resulting $\mathrm{CMB}$ power spectra with data simulated for ideal and Planck experiments using Markov Chain Monte Carlo (MCMC) likelihood analysis as we describe in the next section.

\section{B. Data and Model Optimization}

We use a two-step optimization process to determine the maximum reduction in significance of false positive or negative rejection that can be caused by reionization features. We categorize models in this section by whether $\Delta_{\mathcal{R}}^{2}(k)$ is smooth (S) or has a feature $(\mathrm{F})$, and by whether the reionization history is instantaneous (I) or more complex (C) and parametrized by principal components as in Eq. (23). For comparisons of models we introduce the notation false:true; for example, FI:SI represents the false positive test for instantaneous reionization models from $\S$ III.

In the case of false positives, the goal of the optimization is to go from the FI:SI comparison of the previous section to FC:SC, in which both false and true models have complex ionization histories. In particular, we want to find the FC:SC pair that minimizes the difference between the two models, thus minimizing the significance of false positive rejection. To find this optimal pair of models, we use the following procedure:

1. Optimize true $(S)$ model: $\mathrm{FI}:(\mathrm{SI} \rightarrow \mathrm{SC})$ Vary the ionization history of the SI model to find the SC model that best matches FI.

2. Optimize false $(F)$ model: $(\mathrm{FI} \rightarrow \mathrm{FC}): \mathrm{SC}$

Taking the optimal SC model from step 1 as the true model used to generate simulated data, vary the ionization history of the FI model to find the FC model that matches the best-fit SC.

Then the significance of rejecting false positives including reionization freedom is $-2 \Delta \ln \mathcal{L}_{E E}$ computed for the optimal FC:SC pair, i.e. the maximum likelihood from step 2 .

These steps for the false positive tests are illustrated in Fig. 12 for the ideal experiment. The process for false negatives can be described by simply swapping which models have features and which are smooth $(\mathrm{F} \leftrightarrow \mathrm{S})$.

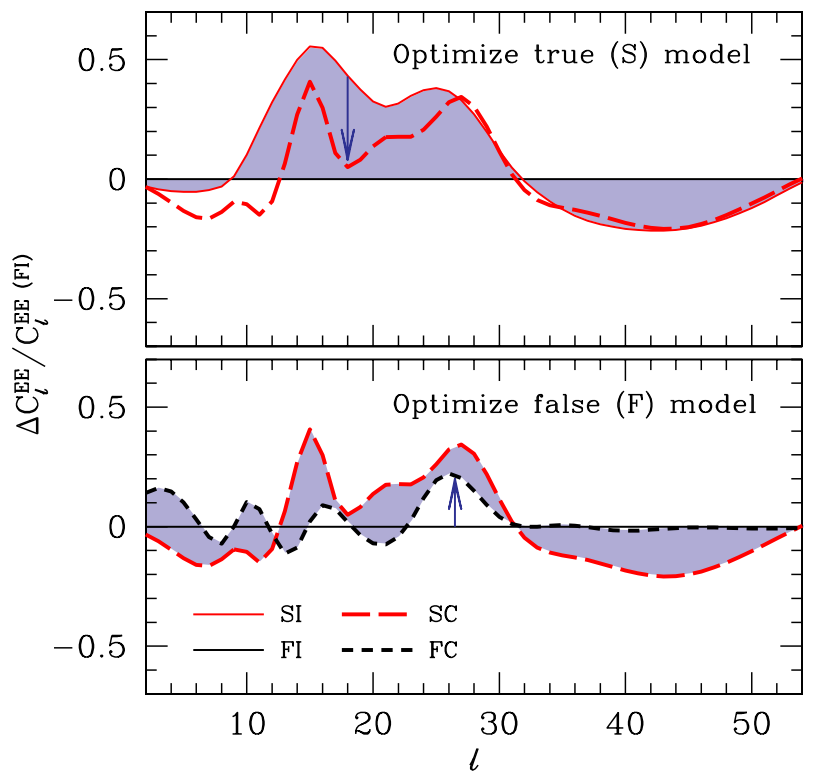

FIG. 12: False positive example of the two-step process to account for reionization uncertainty in polarization significance for the ideal experiment. Upper panel: $\mathrm{FI}:(\mathrm{SI} \rightarrow \mathrm{SC})$ - varying the ionization history from SI to SC (red curves) to match FI (solid black). Lower panel: $(\mathrm{FI} \rightarrow \mathrm{FC}): \mathrm{SC}$ - varying the ionization history from FI to FC (black curves) to match SC (dashed red). All polarization spectra are plotted relative to FI. This joint optimization minimizes the FI:SI difference (shading in upper panel, with fiducial significance given in Table III) at $\ell \lesssim 30$ using the optimal FC:SC models (shading in lower panel; Table V. See $\S$ IVB for an explanation of the notation used here.

Note that even with our conservative choice of $z_{\max }=$ 50, Fig. 12 shows that the main impact of reionization on the polarization spectra is limited to $\ell \lesssim 30$. We will explore the consequences of this restriction to large scales in the following section.

To implement the steps described above, we vary ionization histories to minimize $-2 \Delta \ln \mathcal{L}_{E E}$ following the methods of Refs. [49, 50, 60], using CosmoMC ${ }^{1}$ [62] for MCMC likelihood analysis with a version of CAMB 63. modified to include reionization histories parametrized with principal components as in Eq. (23). For example, in the FI: $(\mathrm{SI} \rightarrow \mathrm{SC})$ step above, we take the FI model as the simulated polarization data and search over ionization histories of the SC model class.

For each optimization step, we run 4 MCMC chains long enough to be well past any initial burn-in phase and stop when the region of parameter space near the best fit is sufficiently well sampled that all 4 chains agree on the maximum likelihood to within $\sim 1 \%$ in $-2 \Delta \ln \mathcal{L}$. Typically this requires computing an initial chain to estimate the covariance matrix of the reionization $\mathrm{PC}$ amplitudes, followed by generating chains with $\sim 10^{4}$ samples each. The optimal true or false model is taken to be the overall

\footnotetext{
1 http://cosmologist.info/cosmomc/
} 


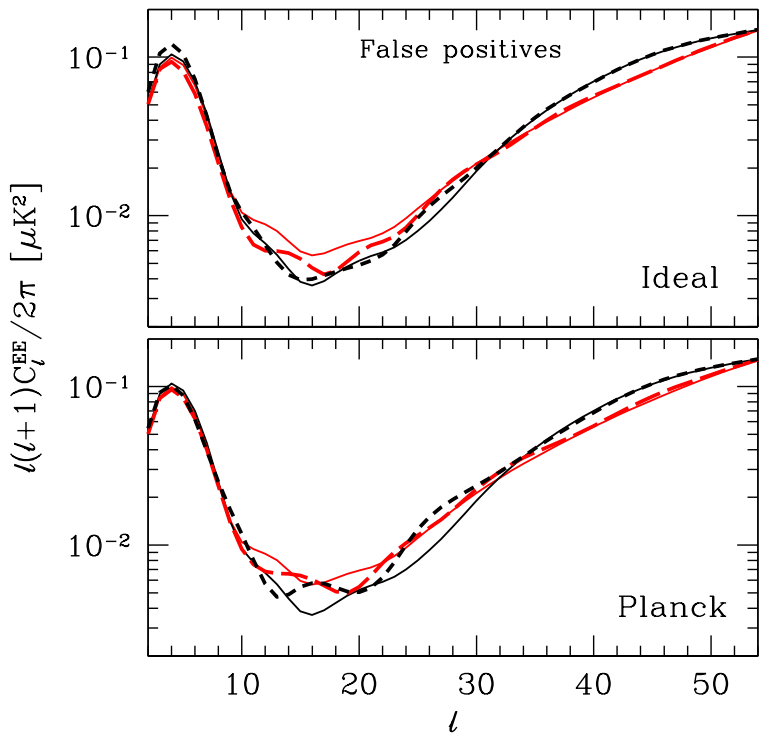

FIG. 13: Test of false positives due to reionization for an idealized experiment limited by sample variance and for Planck. Thick curves show the true smooth model (long dashed red) and bestfit false feature model (short dashed black) for the false positive scenario that would be the most difficult to reject due to freedom in the reionization history. For comparison, the instantaneous reionization polarization spectra from Fig. 4 are plotted as thin solid curves. Reionization histories are parametrized by 7 principal components that cover redshifts $6<z<50$.

maximum likelihood model from the final 4 chains.

All cosmological parameters besides the 7 reionization PC amplitudes are assumed to be fixed by measurements of the temperature spectrum, except for the amplitude of scalar fluctuations $A_{s}$ which is varied to keep $A_{s} \exp (-2 \tau)$ fixed, preserving the temperature and polarization power at small scales. Fixed parameters are set to the values in Table प. We use top-hat priors on the $\mathrm{PC}$ amplitudes corresponding to $0 \leq x_{e} \leq 1$ as described in Ref. [49]. Note that the number of PCs used here is larger than the 3 to 5 needed for completeness in Ref. 49] due to our choice of a larger maximum redshift.

Although we are interested in the ability of polarization data to test features appearing in the observed temperature spectrum, we include the contributions from the model TT and TE spectra as well as EE in the likelihood for MCMC. Keeping the temperature data in the likelihood ensures that we do not obtain models that fit the polarization spectrum well at the expense of changing the shape of $C_{\ell}^{T T}$. For example, ionization histories with sharp transitions in $x_{e}$ at high redshift can generate polarization power at $\ell \sim 40$ to match inflationary features, but these models also add power to the temperature on similar scales through an enhanced Doppler effect [49, 60]. For the best-fit models, the contribution of temperature data to the likelihood is approximately constant: $-2 \Delta \ln \mathcal{L}_{T T} \approx 7$.

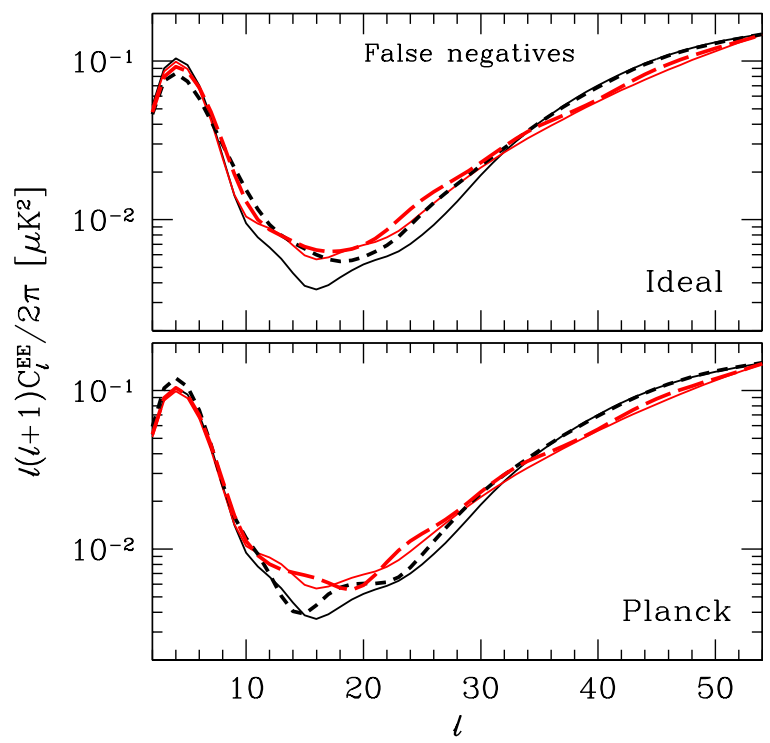

FIG. 14: Same as Fig. 13 but for tests of false negatives. Here the true model has a feature in $\Delta_{\mathcal{R}}^{2}(k)$ and the false model is assumed to have smooth $\Delta_{\mathcal{R}}^{2}(k)(c=0)$.

\section{Reionization Confusion}

The optimal true and false model spectra obtained from the steps described in the previous section (SC and FC) are plotted in Figs. 13 and 14 for each of our 4 scenarios (ideal/Planck tests of false positives/negatives). We also plot the corresponding models with instantaneous reionization histories (SI and FI) to show where ionization freedom has the largest effect on the spectra. Table $\nabla$ lists $-2 \Delta \ln \mathcal{L}_{E E}$ for each FC:SC or SC:FC comparison.

The ability of reionization to either mimic or obscure the signature of inflationary features is greatest in the low-power $10 \lesssim \ell \lesssim 30$ regime of $C_{\ell}^{E E}$. For tests of false positives with an ideal experiment, nearly all of the $25 \%$ reduction of $\sqrt{-2 \Delta \ln \mathcal{L}_{E E}}$ due to reionization comes from $\ell<30$. Planck, on the other hand, has relatively greater sensitivity to small changes in the polarization bump at $\ell \sim 40$ since observations at such scales suffer less from instrumental noise than at $\ell \sim 20$. The $17 \%$ reduction of $\sqrt{-2 \Delta \ln \mathcal{L}_{E E}}$ for false positive rejection for Planck due to reionization is split equally between $\ell<30$ and $\ell \geq 30$. This fact combined with the weakness of reionization effects at $\ell \gtrsim 30$ makes Planck somewhat less sensitive to reionization uncertainties than an idealized noise-free experiment.

For false negative tests, changes to polarization spectra at $\ell>30$ are generally more important than they are for false positives. In the case of the ideal experiment, if we ignored multipoles above $\ell=30$ reionization would only reduce $\sqrt{-2 \Delta \ln \mathcal{L}_{E E}}$ by $20 \%$ relative to the instantaneous reionization significance instead of the $36 \%$ reduction that we find when including all scales. The false 
model being tested in this case ( $\left.\operatorname{smooth} \Delta_{\mathcal{R}}^{2}(k)\right)$ has less power and therefore lower sample variance at $30 \lesssim \ell \lesssim 50$ than the spectrum with a feature, and therefore changes in the polarization spectra on these scales have a greater effect on the significance than they do for false positive tests. Likewise, Planck's significance is more dependent on the $\ell \sim 40$ bump for testing false negatives than for false positives. In fact, nearly all of the $13 \%$ degradation in $\sqrt{-2 \Delta \ln \mathcal{L}_{E E}}$ for false negative rejection comes from $\ell>30$ for Planck.

Changes in the reionization history at $6<z<50$ are unable to significantly affect the polarization power spectrum at $\ell \gtrsim 50$. A detection of polarization features on these scales would therefore be robust to reionization uncertainty. Likewise, measurement of a smooth spectrum on these scales would strengthen bounds on the height and width of a step in the inflaton potential.

By considering variations in $x_{e}(z)$ up to $z=50$, we include a wide variety of ionization histories, many of which may not be physically plausible. In practice, however, the ionization histories of the spectra in Figs. 13 and 14 have $x_{e} \lesssim 0.2$ at $z>20$. Nevertheless, had we chosen to limit ionization variation to lower redshifts the possibility of confusing reionization with inflationary features would be lessened, particularly for tests of false negatives and for Planck, due to the greater reliance on small-scale features in those cases.

Note that the effects of optimizing the ionization history and smoothing the polarization spectra with the addition of a large tensor component ( $\$$ IIC) are similar: both are able to make a spectrum with inflationary features and a smooth spectrum appear more alike at $10 \lesssim \ell \lesssim 30$. Due to this similarity, we expect that considering tensors and reionization simultaneously would not further degrade the significance of false positive or negative tests.

For variations in the potential parameters discussed in $\S$ IIIB that retain only the dip at $\ell \sim 20$ and not the bump at $\ell \sim 40$, the impact of reionization will be greater. In these cases one cannot expect polarization to provide unambiguous confirmation of features without external input on the ionization history.

\section{DISCUSSION}

Models with a step in the inflationary potential produce oscillations in the angular power spectra of the CMB that can improve the fit to WMAP temperature data at multipoles $\ell \sim 20-40$ at the expense of 3 additional phenomenological parameters controlling the a step height, width, and location on the inflaton potential. Such models predict that these oscillations should appear in the $E$ mode polarization spectrum on similar, few-degree scales. The first precise measurements of the polarization on these scales are anticipated in the next few years from Planck, enabling tests of the inflationary-step hypothesis.
Moreover, inflationary features at the upper range of $\ell \gtrsim 30\left(k \gtrsim 2 \times 10^{-3} \mathrm{Mpc}^{-1}\right)$ that are smoothed out due to projection effects in temperature should be more visible in polarization. For the lower range of $\ell \lesssim 30$ $\left(k \lesssim 2 \times 10^{-3} \mathrm{Mpc}^{-1}\right)$, it becomes important to assess the impact of reionization and tensor mode uncertainties.

We have explored in detail the prospects for polarization tests of features, focusing in particular on the risk of errors that can be classified as false positives (falsely confirming an inflationary feature) and false negatives (falsely rejecting an inflationary feature). Under the simplest set of assumptions for large-scale polarization in which we take the best-fit model for the temperature features, neglect tensor fluctuations, and take the reionization history to be instantaneous, polarization measurements from Planck should be able to confirm or exclude the inflationary features that best match current temperature data with a significance of $\sqrt{-2 \Delta \ln \mathcal{L}_{E E}} \sim 3$. All-sky experiments beyond Planck could potentially increase this significance to $\sqrt{-2 \Delta \ln \mathcal{L}_{E E}} \sim 8$, providing a definitive test for features from inflation.

The estimated significance degrades slightly with the addition of a large-amplitude, smooth tensor component to the $E$-mode spectrum, which tends to hide the effect of an inflationary step at the largest scales. Assuming that the step modifies an $m^{2} \phi^{2}$ potential, $\sqrt{-2 \Delta \ln \mathcal{L}_{E E}}$ is reduced by $4 \%$ for Planck and $\sim 14 \%$ for a cosmic variance limited experiment. Allowing non-standard reionization histories with arbitrary changes to the ionized fraction at $6<z<50$ can lower $\sqrt{-2 \Delta \ln \mathcal{L}_{E E}}$ by as much as $\sim 15 \%$ for Planck and $\sim 30 \%$ for cosmic variance limited data. Since tensor fluctuations and reionization have the greatest impact on detectability of inflationary features at similar scales $(\ell \sim 20)$, their effects on the significance should not be cumulative.

The possible contamination due to tensors or reionization could eventually be mitigated with constraints from other types of observations, e.g. stronger limits on the tensor-to-scalar ratio from the $B$-mode polarization power spectrum. The $B$-mode contribution from an $m^{2} \phi^{2}$ potential is potentially within the reach of Planck 64]. Note, however, that a failure to reject false positives or negatives for inflationary features in $E$-mode polarization would generally bias the inferred ionization history and reionization parameters such as the optical depth. Such biases would in turn lead to biased constraints on inflationary parameters from tensor $B$-mode measurements [50].

These estimated significances assume that the parameters of the step in the inflaton potential are those that best fit the WMAP temperature spectrum. Away from this best fit, the polarization significance can either increase or decrease. Cases where the significance substantially decreases correspond to parameter combinations where at most one of the $\operatorname{dip}(\ell \sim 20)$ and bump $(\ell \sim 40)$ temperature features can be explained by the step in the potential. In the case that only the dip is inflationary, Planck will be unable to confirm the feature. 
We have not computed the impact of foreground removal uncertainties on our results; in general one might expect our forecasts to degrade somewhat upon including them. However, recent studies for Planck [64] and a future dedicated polarization satellite mission [65, 66] indicate that foregrounds will not be a substantial problem in the relevant multipole range. Finally, we do not address the possibility that the features in the WMAP data arise from a systematic effect (cf. Appendix $\mathrm{A}$ ). Nonetheless, if all of the $\ell=20-40$ features in the temperature power spectrum are inflationary, polarization should ultimately provide a statistically significant confirmation.

Acknowledgments: We thank Jan Hamann and Richard Easther for valuable conversations. MJM, CD and WH were supported by the KICP through the grant NSF PHY-0114422 and the David and Lucile Packard Foundation. MJM was additionally supported through the NSF GRFP. WH was additionally supported by the DOE through contract DE-FG02-90ER-40560. HVP was supported in part by Marie Curie grant MIRG-CT-2007203314 from the European Commission, and by a STFC Advanced Fellowship. HVP thanks the Galileo Galilei Institute for Theoretical Physics for the hospitality and the INFN for partial support during the completion of this work.

\section{APPENDIX A: RELATION TO PRIOR WORK}

Our best-fit parameters for the WMAP temperature data (Table I) differ from those found by previous studies of the same inflationary model in Refs. 33, 34]. We explain here the reasons for these discrepancies, which include the addition of data and changes in the likelihood code in going from 3-year to 5-year WMAP data, as well as differences in the computation of the evolution of modes during inflation.

Due to small changes in the observed $T T$ spectrum between WMAP 3-year and 5-year data, we find that the best-fit width of the feature increased from $d=0.022$ to a value of $d=0.027$. The lower value of $d$ agrees with the best-fit value found by Ref. [34], which was based on the 3 -year data. Note that a wider feature in $k$ implies a narrower feature in $\ell$ for the CMB power spectra. Fig. 15 shows the best fit models for both data sets along with the appropriately binned data.

Updates to the WMAP likelihood code could also cause small changes in the best-fit potential parameters. In fact, one might be concerned that the feature in the WMAP temperature data is only a systematic effect with some artificial origin in the likelihood calculation. In particular, given the location of a feature, its significance could emerge in some fashion from the transition between the low- $\ell$ pixel-based $T T$ likelihood code and the high- $\ell$ harmonic space likelihood code, which happens at $\ell=32$ in the 5-year likelihood code [3]. However, in the original version of the 3-year likelihood code, v2p1, the transition occurred at $\ell=16$, and in the final version, v2p2p2, it was changed to $\ell=32$ [67]. We searched for the best-fit feature model using WMAP3 data with these two versions of the likelihood code and found almost exactly the same values for the potential parameters in both cases, indicating that this particular issue is not the source of a systematic effect.

Our best fit value for $b$ is considerably different from Refs. [33, 34] even though we use the same matching condition as they do between $e$-folds and physical wavenumbers. This is due to a choice of initial conditions for the background evolution of the inflaton by these authors that did not quite satisfy the Friedmann equation, with the result that the subsequent evolution also failed to satisfy it [68]. This essentially translates into a horizontal shift in $\phi$, changing the preferred location of the step $b$.

Ref. 34] discusses relaxing the model dependence of the predicted power spectrum from the chaotic inflation "toy model" adopted here by using a free spectral index that is fit to the data rather than set by the choice of $N_{\star}$. Since the value of $n_{s} \approx 0.96$ determined by our matching condition for the chaotic inflation potential as described in $\S \coprod$ is nearly identical to the spectral tilt in the WMAP5 best-fit concordance model (i.e. with smooth $\Delta_{\mathcal{R}}^{2}(k)$ ), we do not carry out this extra step here. However, the form of the underlying potential will be tested by the Planck satellite irrespective of the existence of features; as we note in $\S$ IIC the tensor amplitude predicted by the $m^{2} \phi^{2}$ potential (which is not affected by the presence of the feature) is within Planck's reach [64].

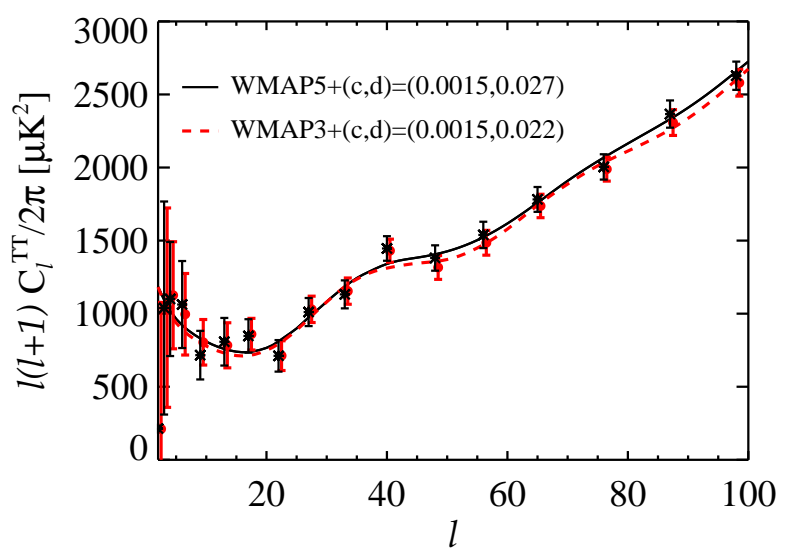

FIG. 15: Observed TT spectrum (binned in $\ell$, with WMAP3 and WMAP5 points offset slightly in $\ell$ for clarity) and best-fit feature models for WMAP3 (red) and WMAP5 (black). Parameters other than $c$ and $d$ are set to the values in Table 1 for WMAP5, and to the following values for WMAP3: $\left\{m=6.852 \times 10^{-6}, b=\right.$ $\left.14.67, \Omega_{b} h^{2}=0.02222, \Omega_{c} h^{2}=0.09927, h=0.753, \tau=0.0817\right\}$.
[1] G. Hinshaw et al., Astrophys. J. Suppl. 180, 225 (2009), arXiv:0803.0732.
[2] C. L. Bennett et al., Astrophys. J. Suppl. 148, 1 (2003), 
astro-ph/0302207.

[3] J. Dunkley et al., Astrophys. J. Suppl. 180, 306 (2009), arXiv:0803.0586.

[4] M. R. Nolta et al., Astrophys. J. Suppl. 180, 296 (2009), arXiv:0803.0593.

[5] E. Komatsu et al., Astrophys. J. Suppl. 180, 330 (2009), arXiv:0803.0547.

[6] H. V. Peiris and R. Easther, JCAP 0807, 024 (2008), arXiv:0805.2154.

[7] W. H. Kinney, E. W. Kolb, A. Melchiorri, and A. Riotto, Phys. Rev. D78, 087302 (2008), arXiv:0805.2966.

[8] L. Alabidi and J. E. Lidsey, Phys. Rev. D78, 103519 (2008), arXiv:0807.2181.

[9] J. Lesgourgues and W. Valkenburg, Phys. Rev. D75, 123519 (2007), astro-ph/0703625.

[10] D. N. Spergel et al., Astrophys. J. Suppl. 170, 377 (2007), astro-ph/0603449.

[11] D. Parkinson, P. Mukherjee, and A. R. Liddle, Phys. Rev. D73, 123523 (2006), astro-ph/0605003.

[12] C. Gordon and R. Trotta (2007), arXiv:0706.3014.

[13] L. Verde and H. V. Peiris, JCAP 0807, 009 (2008), arXiv:0802.1219.

[14] M. Bridges, F. Feroz, M. P. Hobson, and A. N. Lasenby (2008), arXiv:0812.3541.

[15] D. N. Spergel et al., Astrophys. J. Suppl. 148, 175 (2003), astro-ph/0302209.

[16] H. V. Peiris et al., Astrophys. J. Suppl. 148, 213 (2003), astro-ph/0302225.

[17] S. Hannestad, JCAP 0404, 002 (2004), astro$\mathrm{ph} / 0311491$.

[18] A. Shafieloo and T. Souradeep, Phys. Rev. D70, 043523 (2004), astro-ph/0312174.

[19] P. Mukherjee and Y. Wang, Astrophys. J. 599, 1 (2003), astro-ph/0303211.

[20] A. Shafieloo, T. Souradeep, P. Manimaran, P. K. Panigrahi, and R. Rangarajan, Phys. Rev. D75, 123502 (2007), astro-ph/0611352.

[21] G. Nicholson and C. R. Contaldi (2009), arXiv:0903.1106.

[22] A. A. Starobinsky, JETP Lett. 55, 489 (1992).

[23] J. A. Adams, B. Cresswell, and R. Easther, Phys. Rev. D64, 123514 (2001), astro-ph/0102236.

[24] M. Joy, V. Sahni, and A. A. Starobinsky, Phys. Rev. D77, 023514 (2008), arXiv:0711.1585.

[25] J. Silk and M. S. Turner, Phys. Rev. D35, 419 (1987).

[26] R. Holman, E. W. Kolb, S. L. Vadas, and Y. Wang, Phys. Lett. B269, 252 (1991).

[27] D. Polarski and A. A. Starobinsky, Nucl. Phys. B385, 623 (1992).

[28] J. A. Adams, G. G. Ross, and S. Sarkar, Nucl. Phys. B503, 405 (1997), hep-ph/9704286.

[29] P. Hunt and S. Sarkar, Phys. Rev. D70, 103518 (2004), astro-ph/0408138.

[30] J. Lesgourgues, Nucl. Phys. B582, 593 (2000), hep$\mathrm{ph} / 9911447$.

[31] C. P. Burgess, R. Easther, A. Mazumdar, D. F. Mota, and T. Multamaki, JHEP 05, 067 (2005), hepth/0501125.

[32] A. Ashoorioon and A. Krause (2006), arXiv:hepth/0607001.

[33] L. Covi, J. Hamann, A. Melchiorri, A. Slosar, and I. Sorbera, Phys. Rev. D74, 083509 (2006), astro-ph/0606452.

[34] J. Hamann, L. Covi, A. Melchiorri, and A. Slosar, Phys. Rev. D76, 023503 (2007), astro-ph/0701380.

[35] P. Hunt and S. Sarkar, Phys. Rev. D76, 123504 (2007),
arXiv:0706.2443

[36] M. Joy, A. Shafieloo, V. Sahni, and A. A. Starobinsky (2008), arXiv:0807.3334.

[37] J. Martin and C. Ringeval, Phys. Rev. D69, 083515 (2004), astro-ph/0310382.

[38] M. Kawasaki, F. Takahashi, and T. Takahashi, Phys. Lett. B605, 223 (2005), astro-ph/0407631.

[39] R. K. Jain, P. Chingangbam, J.-O. Gong, L. Sriramkumar, and T. Souradeep, JCAP 0901, 009 (2009), arXiv:0809.3915.

[40] W. Hu and T. Okamoto, Phys. Rev. D69, 043004 (2004), astro-ph/0308049.

[41] N. Kogo, M. Sasaki, and J. Yokoyama, Phys. Rev. D70, 103001 (2004), astro-ph/0409052.

[42] C. Pahud, M. Kamionkowski, and A. R. Liddle, Phys. Rev. D 79, 083503 (2009), arXiv:0807.0322.

[43] R. Nagata and J. Yokoyama, Phys. Rev. D79, 043010 (2009), arXiv:0812.4585.

[44] O. Dore, G. P. Holder, and A. Loeb, Astrophys. J. 612 , 81 (2004), astro-ph/0309281.

[45] C. Skordis and J. Silk (2004), astro-ph/0402474.

[46] C. Gordon and W. Hu, Phys. Rev. D70, 083003 (2004), astro-ph/0406496.

[47] W. Fang et al., Phys. Rev. D78, 103509 (2008), arXiv:0808.2208.

[48] W. Hu and G. P. Holder, Phys. Rev. D68, 023001 (2003), astro-ph/0303400.

[49] M. J. Mortonson and W. Hu, Astrophys. J. 672, 737 (2008), arXiv:0705.1132.

[50] M. J. Mortonson and W. Hu, Phys. Rev. D77, 043506 (2008), arXiv:0710.4162.

[51] The Planck Collaboration (2006), astro-ph/0604069.

[52] D. Baumann et al. (2008), arXiv:0811.3919.

[53] C. Dvorkin, H. V. Peiris, and W. Hu, Phys. Rev. D77, 063008 (2008), arXiv:0711.2321.

[54] S. M. Leach and A. R. Liddle, Phys. Rev. D63, 043508 (2001), astro-ph/0010082.

[55] V. F. Mukhanov, Zhurnal Eksperimental noi i Teoreticheskoi Fiziki 94, 1 (1988).

[56] M. Sasaki, Progress of Theoretical Physics 76, 1036 (1986).

[57] E. D. Stewart and D. H. Lyth, Physics Letters B 302, 171 (1993), gr-qc/9302019.

[58] V. F. Mukhanov, JETP Lett. 41, 493 (1985).

[59] V. F. Mukhanov, H. A. Feldman, and R. H. Brandenberger, Phys. Rep. 215, 203 (1992).

[60] M. J. Mortonson and W. Hu, Astrophys. J. Lett. 686, L53 (2008), 0804.2631.

[61] X.-H. Fan, C. L. Carilli, and B. G. Keating, Ann. Rev. Astron. Astrophys. 44, 415 (2006), astro-ph/0602375.

[62] A. Lewis and S. Bridle, Phys. Rev. D66, 103511 (2002), astro-ph/0205436.

[63] A. Lewis, A. Challinor, and A. Lasenby, Astrophys. J. 538, 473 (2000), astro-ph/9911177.

[64] G. Efstathiou, S. Gratton, and F. Paci (2009), arXiv:0902.4803.

[65] L. Verde, H. Peiris, and R. Jimenez, JCAP 0601, 019 (2006), astro-ph/0506036.

[66] J. Dunkley et al. (2008), arXiv:0811.3915.

[67] G. Hinshaw et al., Astrophys. J. Suppl. 170, 288 (2007), astro-ph/0603451.

[68] J. Hamann (2009), private communication. 